\title{
Re: Hydrodistention of the Bladder for the Treatment of Bladder Pain Syndrome/Interstitial Cystitis (BPS/IC)
}

\author{
Hoke TP' ${ }^{1}$ Goldstein $\mathrm{H}^{2}$, Saks EK², Vakili B ${ }^{2}$ \\ ${ }^{1}$ Christiana Care Health System, Clinic of Obstetrics and Gynecology, Newark, USA \\ ${ }^{2}$ Christiana Care Health System, Center for Urogynecology and Pelvic Surgery, Newark, USA
}

Neurourol Urodyn 2017;36:784-786. doi: 10.1002/nau.23024.

\section{EDITORIAL COMMENT}

Despite there are many causes of bladder pain syndrome/interstitial cystitis (BPS/IC) in theory, the real etiology is still obscure. Life quality of patients is already deteriorated when this syndrome is diagnosed by eliminating other causes. Usually, a single therapy is insufficient. The aim of treatment is to relieve the symptoms.

Cystoscopy with hydrodistention is a longstanding investigation used in both diagnosis and treatment. The results of cystoscopy with hydrodistention treatments are frequently empirical. The mechanism of action is explained by disruption of the bladder sensory nerves. Although efficacy is considerable in a group of patients in early period, it decreases by time. Use of trigonal block anecdotally before hydrodistention is known to be more effective than hydrodistention alone.

In this study, hydrodistention at a pressure of $80 \mathrm{~cm}$ was performed with a distention time dichotomized into 2 and $>5$ min in patients with suitable indications. Seventy-seven of 183 patients who underwent hydrodistention were excluded. Of the remaining 106 patients, 48 received hydrodistention with prior trigonal block and 58 patients only hydrodistention. While the primary outcome of the study was change in pain, secondary outcomes were to measure narcotics usage, need for further treatment and status of symptoms. Time of distention was also compared. While there was a significant improvement in pain in both groups, no significant difference was found in pain scores between the two groups. Time of hydrodistention was found to be significantly longer in the group of patients in whom trigonal block was performed.

BPS/IC is a distressing condition for patients affected. Defining the etiology and development of treatment modalities are essential. Hydrodistention is an effective treatment method in certain group of patients. Thus, if cystoscopy is performed in any circumstances, benefits of hydrodistention must be kept in mind.

İlker Şen, MD 